\title{
Application of Local Binary Pattern to Windowed Nonlocal Means Image Denoising
}

\author{
Fakhry Khellah \\ Prince Sultan University, Riyadh - 11586, Saudi Arabia \\ fkhellah@cis.psu.edu.sa
}

\begin{abstract}
This paper presents a new technique to image denoising that mainly addresses the incurred high blurring when the windowed nonlocal means is applied to images corrupted by high noise levels. The proposed method is based on an enhanced weighting function that computes patches similarity based on both their intensities and structural features. The structural features are encoded using Local Binary Pattern (LBP) a well known texture descriptors. A new LBP based weighting function is proposed that has properties complementing the intensity based weighting function. The LBP based weighting function is used to modulate the intensity based weighting function. The modulated weights are noise independent and reflect the actual patch similarity. The method is found to be quantitatively and qualitatively effective in denoising images when corrupted by high noise levels. It suppresses image noise while preserving significant image characteristics.
\end{abstract}

Keywords: Local Binary Patterns, Nonlocal means, Image Denoising.

\section{Introduction}

Image denoising is still one of the most fundamental areas of research in the field of image processing. Some of the spatial domain methods restore the intensity value of each image pixel by averaging in some way the intensities of its nearby neighbors. The major drawback of such techniques is that they blur the small scale structures in the image such as edges or textures. One of the introduced spatial domain methods that has been shown to produce high quality results is the nonlocal means filtering method proposed by Buades [2]. The nonlocal means algorithm is based on the idea that natural and textured images have redundancy and any image pixel has similar pixels that are not necessarily located in a nearby spatial neighborhood. In the classical definition of the nonlocal means filter, for each pixel being processed in the image, the whole image is searched and differences between corresponding neighborhoods (patches) are computed. In [2], the weights computation was limited to a local subdomain called search window centered at the pixel being processed. The method is called windowed nonlocal means filter. One main drawback of the nonlocal means filter is the obvious blurring of edges and fine texture details incurred to denoised images

A. Petrosino (Ed.): ICIAP 2013, Part I, LNCS 8156, pp. 21-30 2013.

(C) Springer-Verlag Berlin Heidelberg 2013 


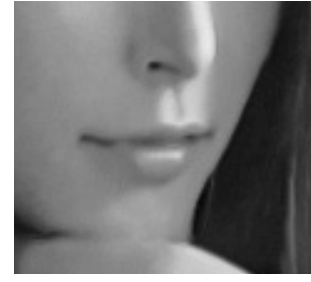

Denoised $(\sigma=10)$

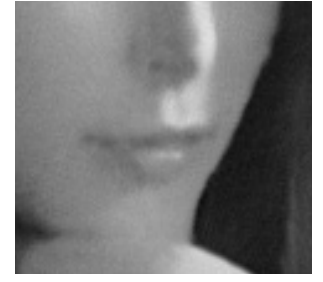

Denoised $(\sigma=25)$

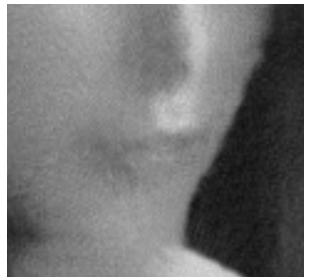

Denoised $(\sigma=50)$

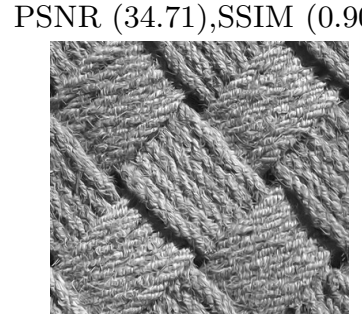

Denoised $(\sigma=10)$

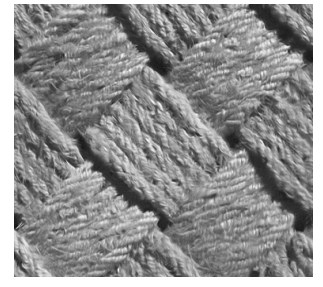

Denoised $(\sigma=25)$

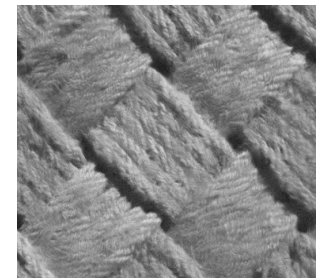

Denoised $(\sigma=50)$

PSNR (24.53),SSIM (0.90) PSNR (20.65),SSIM (0.71) PSNR (17.66),SSIM (0.45)

Fig. 1. Illustration of effect of the noise level on the windowed nonlocal means filter denoising quality. The smoothness increases as the noise level increases. The top row is zoomed Lena image, and the bottom row is zoomed D1 image.

especially at high noise levels. This is due to incorporating exaggerated weights that do not reliably reflect the actual patch similarity in the averaging of a given pixel. As a result, at high noise levels, the restored pixel will be approximately the average of the intensity values of almost all the pixels in the search window leading to strong smoothing of the image, and therefore, blurring of small scale structures of the image. Particularly, in textured regions of an image, small scale structures are not necessarily equivalent to noise. This is shown in Fig. 1 where the windowed nonlocal means filter is used to denoise two different types of images: a natural image; the standard Lena image, depicted in the top row of Fig. 1 and a highly textured image, (D1) depicted in the bottom row of the figure. The images were corrupted by three noise levels: $(\sigma=10,25,50)$. Observe the high blurring effect as the noise level increases.

In this work, we address the above drawback of the windowed nonlocal means filter by incorporating the structural features of the region where the restored pixel is located in the computation of its averaging weights.

The rest of the paper is organized as follows. In Section 2 an overview of the windowed nonlocal means image denoising method is presented. The proposed filtering method using is described in Section 3. Experimental results are presented and discussed in Section 4. Finally, conclusions are given in Section 5 . 


\section{Overview of the Windowed Nonlocal Means Filter}

In this section, we present a brief overview of the nonlocal means method introduced in 2]. Let the observed noisy intensity of pixel $i$ be defined as: $I_{n}(i)=I_{o}(i)+n(i)$, where $I_{o}(i)$ is the original intensity of the noise-free pixel $i$ and $n \sim \mathcal{N}\left(0, \sigma^{2}\right)$ is the observation noise. In the original formulation of the nonlocal means filter, the restored intensity $\hat{I}(i)$, is the weighted average of all pixel intensities in the noisy image $I_{n}(i)$ defined as:

$$
\hat{I}(i)=\sum_{j \in I_{n}} w(i, j) I_{n}(j)
$$

The weight $w(i, j)$ quantifies the similarity between two square local neighborhoods (patches) $N_{i}$ and $N_{j}$ centered on pixels $i$ and $j$ of predefined size $r \times r$ with typical sizes of $7 \times 7$ or $9 \times 9$. In order to reduce the computational complexity, the sum in (11) is restricted to pixels within a local subdomain around pixel $i$ called search window $S_{i}$ of size $M \times M$ with a typical size of $21 \times 21$ [1], [2, [3], [7]. This version of the nonlocal means filter is usually called semilocal or windowed nonlocal means. For each pixel $i$ the weight in (11) is computed by:

$$
w(i, j)=\frac{1}{Z(i)} e^{-\frac{\left\|v\left(N_{i}\right)-v\left(N_{j}\right)\right\|_{2, a}^{2}}{h^{2}}}
$$

Where $Z_{i}$ is a normalizing term ensuring that all computed weights will add to one, i.e., $\Sigma_{j} w(i, j)=1$. The nonlocal means filter computes the intensity similarity between any two image pixels $i$ and $j$ based on their local neighborhoods $N_{i}$ and $N_{j}$ rather than their individual values. In the above equation, $v\left(N_{i}\right)$ is the vector of neighborhood pixel values around pixel $i$. The vector norm in (2) computes Euclidean distance weighted by a Gaussian kernel of standard deviation $a$ between neighborhood intensities $N_{i}$ and $N_{j}$.

The exponential weighting function in (1) is controlled mainly by two parameters: the intensity based similarity criterion and the smoothing kernel width parameter $h$; that controls the extent of averaging. Both parameters contributes directly to the filter performance degradation as the noise level increases.

The similarity criterion measures the similarity between two patches based on their intensities regardless of their underlying textured or structural characteristics such as curves, edges, corners, and spots. As a fact, two neighboring patches that are similar with respect to their measured intensities according to (1D) are not necessarily similar with respect to their primitive textured structures. This is evident in texture images and in textured regions of natural images. Consequently, image details and fine texture can be oversmoothed due to incorporating dissimilar patches in the denoising of a given pixel.

The smoothing parameter $h$ of the weighting function controls the extent of averaging and is usually set to the noise standard deviation $h=\sigma$ [2] regardless of the smoothness degree of the region where the search window $S_{i}$ is located. Consequently, when the noise standard deviation is high, the smoothing parameter $h$ will be also high and therefore, the exponential weighting function (2) is 


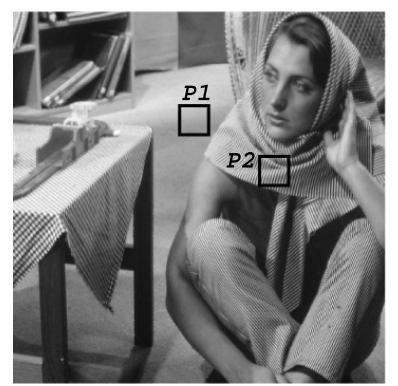

(a) Barbara image showing the location of two pixels $P 1$ and $P 2$ located in flat and textured regions, respectively.

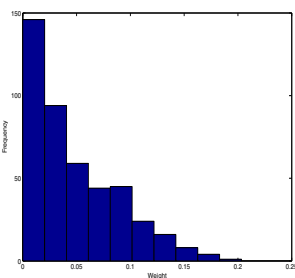

(b) $(\sigma=10)$

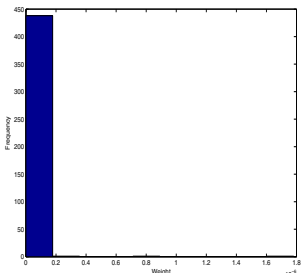

(e) $(\sigma=10)$

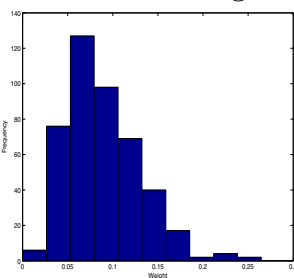

(c) $(\sigma=25)$

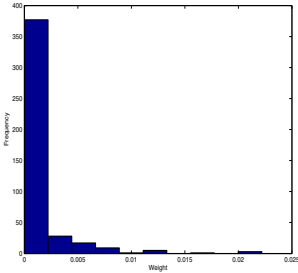

(f) $(\sigma=25)$

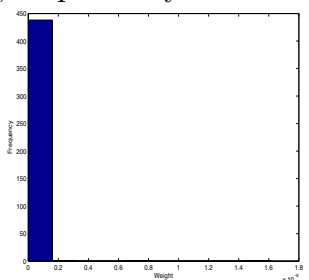

(d) $(\sigma=50)$

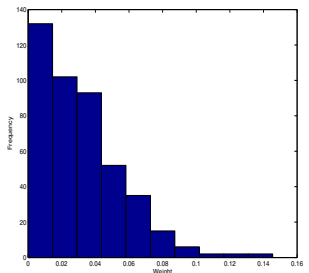

(g) $(\sigma=50)$

Fig. 2. Histograms of the intensity based weights for two Barbara image pixels $P 1$ (top row), and $P 2$ (bottom row) located in smooth and textured regions, respectively

mainly dominated by $h$. Therefore, the computed weights will not exactly reflect the similarity of the involved patches. Consequently, exaggerated weights will be assigned to almost all patches within the search window $S_{i}$. As a result, the restored value $\hat{I}(i)$ will be approximately the average of the intensity values of all the pixels in the search window $S_{i}$ leading to strong smoothing of the image, and therefore, blurring of small scale structures of the image.

The above issues related to the degradation in the filter performance are illustrated in Fig. 2 which depicts the histograms (distributions) of the computed intensity based similarity weights for all patches within a $21 \times 21$ search window centered at two pixels of the standard Barbara image Fig. 2(a): $P 1$ located in a flat region (the top row of Fig. 2) and P2 located at textured region (the bottom row of Fig. 2). The weights are computed for three different noise standard deviations $(\sigma=10,25,50)$. First, observe that for the two regions, as the noise level increases, more dissimilar patches are incorporated and considered to be 
significant. This is clearly reflected in the computed histograms as they become flatter. For example, compared to the histograms in Figs. 2(b,e) when $(\sigma=10)$ for both pixels, the histograms in Figs. $2(\mathrm{~d}, \mathrm{~g})(\sigma=50)$ are much wider and there is a clear reduction in number of eliminated patches (the one assigned approximately zero weights) and increase in the number of patches that are considered significant (assigned high weights). More dissimilar patches to the reference patch are incorrectly incorporated while there has been no change in the structure of the underlying patches to be considered similar to the central patch.

In order to address the aforementioned issues that cause high blurring in the denoised image and becomes highly apparent as the noise level increase, we propose refining the filter intensity based weighting function using a new weighting function that is independent of the noise and utilizes a similarity selection criterion based on the patches structural characteristics. This is detailed in the following section.

\section{LBP Based Nonlocal Means Filter}

The proposed approach for enhancing the nonlocal mean filter is based on modulating the intensity based weights computed in (2) using another weighting function that computes the similarity between any two patches using their textured and structural features obtained by the LBP framework. The modulated weights $w_{m}(i, j)$ are obtained as a product of both the intensity based weights and the proposed LBP based weights:

$$
w_{m}(i, j)=w(i, j)_{\text {intensity }} * w(i, j)_{\mathrm{LBP}}
$$

A brief overview of the LBP feature extraction method is presented in the following section as detailed in [5], 6],

\subsection{LBP Overview}

LBP is a gray-scale texture operator which characterizes the spatial structure of the local image texture. At a center pixel, each neighboring pixel is assigned a binary label, which can be either 0 or 1 , depending on whether the center pixel has higher intensity value than the neighboring pixel. The neighboring pixels are the angularly evenly distributed sample points over a circle with radius $R$ centered at the center pixel. The LBP label for that center pixel is given by [5]:

$$
\operatorname{LBP}_{P, R}=\sum_{p=0}^{P-1} s\left(g_{p}-g_{c}\right) 2^{p}
$$

where $g_{c}$ is the gray value of the central pixel, $g_{p}$ is the value of its $p^{t h}$ neighboring pixel, $p=0, \cdots, P-1, P$ is the total number of neighbors, and $R$ is the radius of 
the neighborhood which determines how far the neighboring pixels are located away from the center pixel. $s(x)$ is a step function given by:

$$
s(x)=\left\{\begin{array}{l}
1, x \geq 0 \\
0, x<0
\end{array}\right.
$$

The value of $P$ is assigned according to the value of $R$ as suggested in 6]. For example, $P=8$ when $R=1, P=16$ when $R=2$, and $P=24$ when $R=3$. The total number of possible LBP features depends on $P$ and is equal to $2^{P}$. For the purpose of image denoising, we adopt certain class of local binary texture patterns termed "uniform" which have limited number of transitions or discontinuities in the circular presentation of the pattern. The "uniform" binary patterns correspond to primitive microfeatures, such as edges, corners, and spots [6]. The total number of possible "uniform" LBP features is $P *(P-1)+2$ [6]. In texture classification framework, given an image $I$ of size $N \times M$, the LBP pattern is identified for each pixel $i$. Then, the whole image is represented by building a histogram $\mathrm{H}$ of the generated LBP features:

$$
\begin{gathered}
H_{k}(I)=\sum_{i=1}^{N} \sum_{j=1}^{M} f(\operatorname{LBP}(i), k), k \in[0, K] \\
f(x, y)=\left\{\begin{array}{l}
1, x=y \\
0, \text { otherwise }
\end{array}\right.
\end{gathered}
$$

Where $K$ is the maximal LBP pattern value.

In this work, the LBP histogram is computed for a square patch $L$ of size $l \times l$ surrounding each individual image pixels.

Therefore, the proposed LBP based weighting function is given by:

$$
w(i, j)_{\mathrm{LBP}}=\frac{1}{Z(i)} e^{-\frac{D\left(H\left(L_{i}\right)-H\left(L_{j}\right)\right)}{h\left(S_{i}\right)}}
$$

Where $Z_{i}$ is a normalizing term, and $H\left(L_{i}\right)$ and $H\left(L_{j}\right)$ are the histograms of the extracted LBP features of the two patches $L_{i}$ and $L_{j} . D$ refers to the Chi-square dissimilarity metric used to compare histograms and given by [6]

$$
D\left(H\left(L_{i}\right), H\left(L_{j}\right)\right)=\sum_{n=1}^{B} \frac{\left(H\left(L_{i}\right)_{n}-H\left(L_{j}\right)_{n}\right)^{2}}{H\left(L_{i}\right)_{n}+H\left(L_{j}\right)_{n}}
$$

Where $B$ is the number of bins and $H\left(L_{i}\right)_{n}$ and $H\left(L_{j}\right)_{n}$ are, respectively, the values at the $n^{\text {th }}$ bin. Unlike the smoothing parameter $h$ of the intensity based weighting function which is set globally (i.e., for the whole image), the decay parameter $h\left(S_{i}\right)$ of the LBP based exponential weighting function in (8) varies according to the smoothness of the underlying region where the search window $S_{i}$ is located and centered by the reference pixel $i$. The region smoothness is estimated from the sampled standard deviation of all the LBP similarity distances obtained according to (9) between the reference patch centered by the noisy pixel $i$ and all other patches within the search window $S_{i}$. 


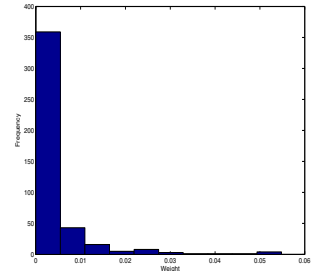

(a) $(\sigma=10)$

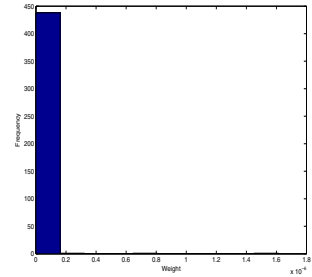

(d) $(\sigma=10)$

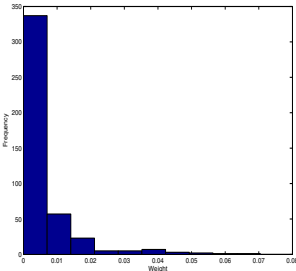

(b) $(\sigma=25)$

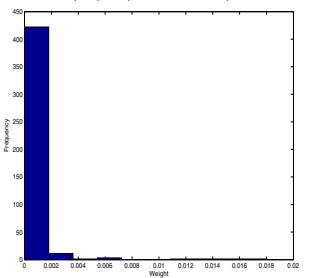

(e) $(\sigma=25)$

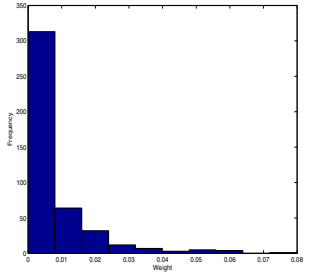

(c) $(\sigma=50)$

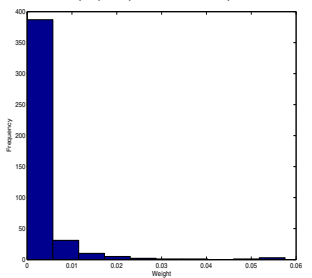

(f) $(\sigma=50)$

Fig. 3. Histograms of the modulated weights for the two Barbara image pixels $P 1$ (top row), and $P 2$ (bottom row) located in smooth and detailed regions, respectively

The proposed LBP based weighting function in (8) poses two important properties: being noise independent and producing weights that vary according to structural characteristics of the underlying region. The two properties of the LBP based weighting function complement the intensity based weighting function once combined together as in (3). This is illustrated in Fig. 3 which shows the distribution of the modulated weights $w_{m}(i, j)$ for the same two pixels from the Barbara image Fig. 2(a): $P 1$ and $P 2$. Observe that the distribution of the modulated weights does not vary as the noise level increases. In other words, the number of patches that are considered significant and assigned high modulated weights stay the same regardless of the noise level. When comparing the modulated weight histograms Fig. 3 for both pixels, we found that they are almost identical to the intensity based histograms generated when the noise level is low $(\sigma=10)$ Fig. $2(\mathrm{~b}, \mathrm{e})$. That is, at all noise levels, the modulated weights will always be equivalent to the most reliable intensity based weights generated when the noise level is low. This shows that the generated weights are noise independent and, hence, are more reliable in reflecting the actual patch similarity regardless of the noise level. The effect of having region based smoothing parameter $h\left(S_{i}\right)$, can be observed when comparing the intensity based weights histogram of pixel P1 Fig. $2(\mathrm{~b})(\sigma=10)$ and the modulated weights histograms, for all noise levels depicted at the top row of Fig. 3, In case of pixels located in flat regions, $h\left(S_{i}\right)$ will be low. Therefore, the LBP based weighting function will be more selective and will have the effect of scaling down the intensity based weights. Consequently, only few patches in flat regions will be considered significant and assigned high modulated weights. This has the effect of reducing the resulting blurring especially in flat regions. 


\subsection{Implementation Details}

The proposed LBP based nonlocal means filter is performed in two main stages. In the first stage, histograms for "uniform" LBP features using $R=2$ and $P=16$ are computed for all image pixels and stored in a lookup table. In this work, the sufficient size of the LBP patch $L$ is determined experimentally and found to be $13 \times 13$. During the filtering stage of a given image pixel $i$, both intensity based weights and LBP based weights are computed according to (2), (8), respectively. Then, modulated weights are computed according to (3).

\section{Experimental Results}

A qualitative comparison using the peak signal-to-noise ratio (PSNR) between the proposed method and the intensity based windowed nonlocal means filter is presented in Table 1. The experiments are conducted on five standard test images using three noise levels $(\sigma=10,25,50)$. The results show that the proposed method produces higher PSNR than the intensity based windowed nonlocal means for all test cases. Visual comparisons between the intensity based windowed nonlocal means filter and the proposed method are shown in Fig. 4 and Fig. 5. Comparing the proposed method results depicted in Fig. 4 to the denoised images by the intensity based windowed nonlocal means filter shown in Fig. 1, it is obvious that the proposed method produces images with noticeably better visual quality. This is supported by the higher structural similarity index (SSIM) values obtained by the proposed method. The blurring effect of the filter is highly reduced especially at high noise levels (i.e., $\geq=25$ ). In case of

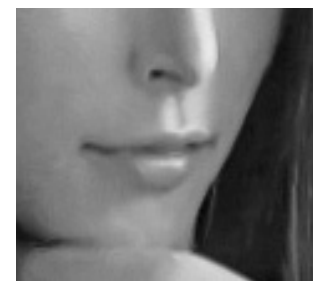

Denoised $(\sigma=10)$
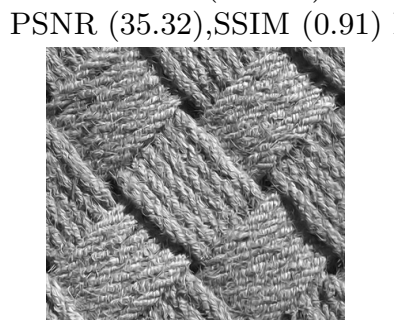

Denoised $(\sigma=10)$

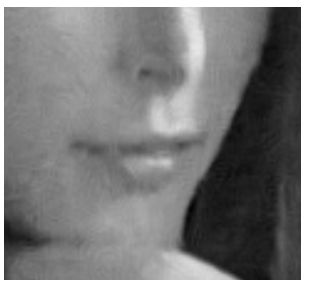

Denoised $(\sigma=25)$ PSNR (31.05),SSIM (0.82) PST

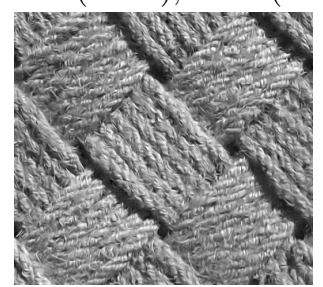

Denoised $(\sigma=25)$

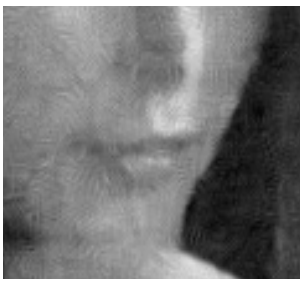

Denoised $(\sigma=50)$
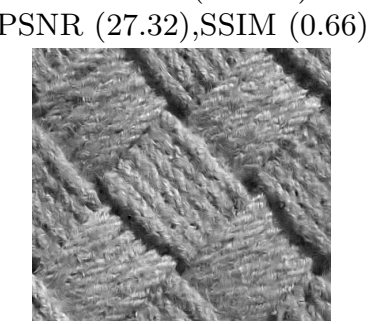

Denoised $(\sigma=50)$

PSNR (24.47),SSIM (0.90) PSNR (21.70),SSIM (0.78) PSNR (18.79),SSIM (0.55)

Fig. 4. PSNR and SSIM for Denoised images using the proposed method 


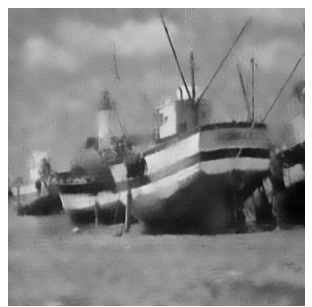

(a)

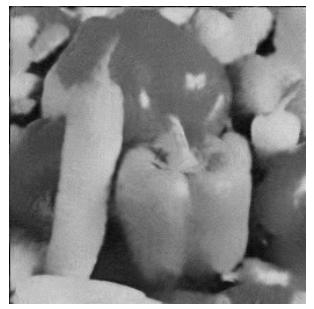

(c)

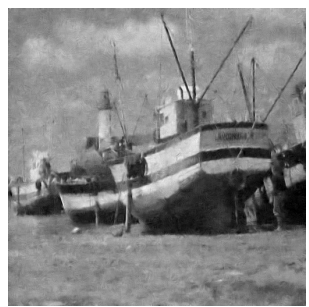

(b)

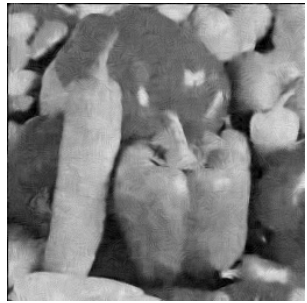

(d)

Intensity Based Proposed method

Nonlocal means

Fig. 5. Comparison of the denoised images using the proposed method and the intensity based Windowed nonlocal means filter. The noise standard deviation is 50 .

Table 1. Comparison of the denoising quality in terms of PSNR for images denoised with the proposed method and the intensity based windowed nonlocal means for five standard test images. For each image, the three rows correspond to noise levels $(\sigma=$ $10,25,50)$.

\begin{tabular}{|c|c|c|c|}
\hline \multirow{4}{*}{$\begin{array}{c}\text { Broposed } \\
\text { Method } \\
\text { PSNR (dB) }\end{array}$} & $\begin{array}{c}\text { Windowed } \\
\text { Nonlocal Means } \\
\text { PSNR (dB) }\end{array}$ & $\begin{array}{c}\text { PSNR } \\
\text { Gain }\end{array}$ \\
\cline { 2 - 4 } & 34.00 & 33.69 & +0.31 \\
\cline { 2 - 4 } & 28.93 & 28.34 & +0.59 \\
\hline \hline Boat & 24.75 & 24.24 & +0.51 \\
\cline { 2 - 4 } & 33.13 & 32.38 & +0.75 \\
\cline { 2 - 4 } & 29.02 & 27.74 & +1.28 \\
\hline \hline House & 25.46 & 24.45 & +1.01 \\
\cline { 2 - 4 } & 35.66 & 35.29 & +0.37 \\
\cline { 2 - 4 } & 31.46 & 30.85 & +0.61 \\
\hline \hline Lena & 27.12 & 26.22 & +0.90 \\
\cline { 2 - 4 } & 35.32 & 34.71 & +0.61 \\
\cline { 2 - 4 } & 31.05 & 30.13 & +0.92 \\
\hline \hline Peppers & 27.32 & 26.68 & +0.64 \\
\cline { 2 - 4 } & 29.55 & 33.05 & +0.50 \\
\cline { 2 - 4 } & 25.60 & 24.43 & +1.00 \\
\hline \hline
\end{tabular}


texture image (D1) depicted in the second row of Fig. 4, the proposed method is able to preserve fine texture details while removing undesirable noisy artifacts. The effect of having region dependent filter smoothing parameter on the visual quality of denoised flat image regions is illustrated in Fig. 5. The denoised flat regions using the proposed method (such as the sea and the pepper surfaces) Fig. 5(b,d) are not oversmoothed and have even clearer fine details than the denoising results of the intensity based windowed nonlocal means filter (Fig. 5)(a,c)).

\section{Conclusions}

This paper introduced a novel approach to nonlocal means image denoising that computes the filter averaging weights using not only patch intensities but also their structural features extracted by the LBP method. Experimental results show that the proposed method has the advantage of preserving high frequency components and suppressing undesirable artifacts such as noise even when the noise level is high. The proposed LBP based windowed nonlocal means outperforms the intensity based windowed nonlocal means algorithm both quantitatively and qualitatively.

\section{References}

1. Brox, T., Kleinschmidt, O., Cremers, D.: Efficient nonlocal means for denoising of textural patterns. IEEE Trans. Image Process. 17(7), 1083-1092 (2008)

2. Buades, A., Coll, B., Morel, J.M.: A non-local algorithm for image denoising. In: International Conference on Computer Vision and Pattern Recognition, pp. 60-65 (2005)

3. Kervrann, C., Boulanger, J.: Optimal spatial adaptation for patch-based image denoising. IEEE Trans. Image Process. 15, 2866-2878 (2006)

4. Liao, S., Law, M.W.K., Chung, A.C.S.: Dominant local binary patterns for texture classification. IEEE Trans. Image Process. 18, 1107-1118 (2009)

5. Liao, S., Law, M.W.K., Chung, A.C.S.: Dominant local binary patterns for texture classification. IEEE Trans. Image Process. 18(5), 1107-1118 (2009)

6. Ojala, T., Pietikainen, M., Maenpaa, T.: Multiresolution gray-scale and rotation invariant texture classification with local binary patterns. IEEE Trans. Pattern Anal. Mach. Intell. 24(7), 971-987 (2002)

7. Tasdizen, T.: Principal neighborhood dictionaries for nonlocal image denoising. IEEE Trans. Image Process. 18(12), 2649-2660 (2009) 